\title{
Complications of Mandibular Fracture Repair and Secondary Reconstruction
}

\author{
Daniel Perez, DDS ${ }^{1} \quad$ Edward Ellis III, DDS ${ }^{1}$ \\ ${ }^{1}$ Department of Oral and Maxillofacial Surgery, University of Texas \\ Health Science Center at San Antonio, San Antonio, Texas
}

Semin Plast Surg 2020;34:225-231.

\begin{abstract}
Address for correspondence Edward Ellis III, DDS, Department of Oral and Maxillofacial Surgery, University of Texas Health Science Center at San Antonio, 8210 Floyd Curl Dr. MC8124, San Antonio, TX 78229-3923 (e-mail: ellise3@uthscsa.edu).
\end{abstract}

\author{
Abstract \\ Keywords \\ - complications \\ - mandible \\ - fractures
}

Mandibular fractures are common facial injuries. Their treatment varies as do postoperative complications. This paper discusses the common complications that are associated with the treatment of mandibular fractures and presents management strategies.
The most common complication cited in the literature after treatment of a mandibular fracture is infection followed closely by malunion. ${ }^{1}$ Many surgeons agree that preoperative infection, greater displacement of the fragments, and the presence of multiple fractures and comorbidities increase the risk of complications. ${ }^{1-4}$

The purpose of this article is to present and discuss the most common complications a surgeon can face when treating mandibular fractures and to delineate their causes and management. A corollary is to provide guidance to decrease the incidence of complications.

\section{Classification of Complications}

One can classify mandibular fracture complications into three main groups: complications related to the fracture location (anatomical), complications related to the internal fixation devices, if placed, and complications related to the patient.

\section{Anatomical Complications}

Complications vary with the location of the fracture. These have to do with the surgical approach(es), the part of the mandible that is affected, and its surrounding structures and/or biomechanics. Any time an extraoral approach is needed, for example in the open treatment of a subcondylar fracture, cranial nerve $(\mathrm{CN})$ VII is at risk of damage; when a symphysis fracture requires intraoral repair, this approach typically would spare CN VII but might affect CN V. Some areas are more difficult to access and repair, which, in turn, increases surgical time, infection rates, and improper application of plates and screws.

\section{Hardware-Related Complications}

Here we can find material wear and fatigue leading to material breakage, hypersensitivity, and allergic reactions, as well as improper selection or application of fixation.

\section{Host-Related Complications}

Patients are all different; their compliance with instructions, their systemic underlying conditions, and the timing of repair all may directly affect how they heal. Complications usually increase with comorbidities and age. ${ }^{2-4}$ They increase with surgical times and length of hospitalization. ${ }^{5}$ The population most associated with mandibular fractures frequently exhibits poor compliance and unwillingness to perform appropriate home care, with a high incidence of substance abuse and inadequate nutrition, increasing the potential problems after surgery. ${ }^{6}$

\section{Factors Associated with Complications}

\section{Anatomical Location of the Fracture}

According to Lamphier et al, the mandibular angle continues to have the highest overall complication rate $(19 \%) .{ }^{6}$ Haug and Schwimmer ${ }^{7}$ showed a $3.2 \%$ incidence of nonunion after
Issue Theme Complications and Secondary Management and Repair in Head, Neck and Plastic Surgery; Guest Editor: Yadranko Ducic, MD, FRCS(C), FACS
Copyright $\odot 2020$ by Thieme Medical Publishers, Inc., 333 Seventh Avenue, 18th Floor, New York, NY 10001, USA Tel: +1(212) 760-0888.
DOI https://doi.org/ $10.1055 / \mathrm{s}-0040-1721758$. ISSN 1535-2188. 
mandibular fractures, with the body of the mandible being the most common site. Bochlogyros ${ }^{8}$ reported only a $0.8 \%$ incidence of nonunions over a 20 -year period, but $51.7 \%$ of their nonunions occurred in the mandibular angle region and $34.4 \%$ in the body.

In the condylar region, infection and nonunion are rare, but injury to the facial nerve is more prevalent than in any other area of the fractured mandible when open treatment is selected.

\section{Duration between Injury and Repair}

Traditionally, it was believed that mandibular fractures should be addressed within 24 to 48 hours, ${ }^{9,10}$ but other studies have shown that further delay does not increase the risk of complications. ${ }^{11-13}$ Logic dictates that whenever possible one should try to treat the fracture as soon as possible. However, patients do not always present immediately after the fracture occurs. Some patients have multiple injuries or comorbidities that need to be treated before one can safely repair their facial fractures. Fortunately, delaying treatment of the mandibular fracture does not doom the patient to complications. James et al found that delays in the treatment of mandibular fractures are not significantly correlated with the development of postoperative complications. A systematic review of 6 studies by Hermund et $\mathrm{al}^{14}$ found no strong evidence for potential benefits of immediate or delayed treatment.

\section{Open versus Closed Treatment of Fracture}

Closed treatment of mandibular fractures tends to have the least amount of complications reported in most studies. ${ }^{15}$ This is true in part because usually the simplest, least displaced fractures are managed closed but also because all the risks and complications associated with the surgical approach(es) and application of fixation devices are not present.

\section{Complications}

\section{Malunion and Malocclusion}

Malunion is defined as the osseous union of a fracture in an incorrect position. The area is healed with bony continuity but there are functional and possibly esthetic problems because the reduction was inadequate. Most postoperative malocclusions are caused by malunions and are usually obvious to both the patient and the surgeon. When the degree of displacement of the healed segments is great, facial deformity may also be noted (-Fig. 1).

The most common causes of malunion are inadequate dental reduction during surgery, inadequate osseous reduction during surgery, imprecise application of internal fixation devices, and/or inadequate stabilization. ${ }^{16}$ Malunions can occur with closed treatment as well. However, the improper use of rigid internal fixation devices can very easily cause it. Improper bending of a plate, inadequate occlusal reduction due to loss of
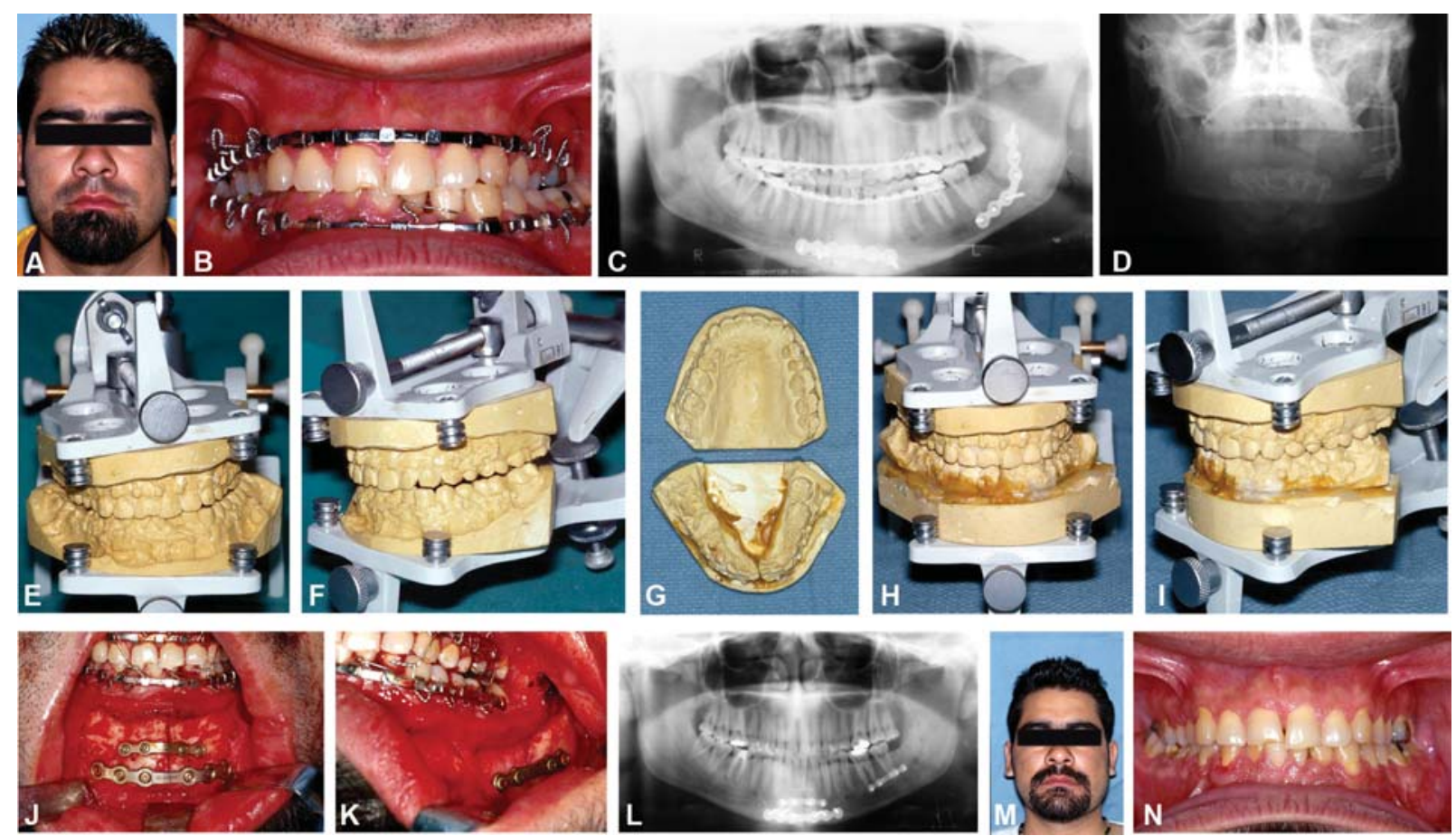

Fig. 1 Records of a patient who was treated elsewhere for left angle and symphysis fractures of the mandible. He complained that his bite was off and that his face looked asymmetric. Additionally, he said he had no feeling in the left lower lip and chin. (A) Facial photograph showing more fullness on the left side of his face. (B) Photo of his occlusion showing left crossbite. (C) Panoramic radiograph showing bone plates attached to the mandible in the symphysis and angle regions. Note the three lower screws through the bone plate at the angle are directly over the inferior alveolar canal. (D) Posteroanterior radiograph showing lateral displacement of the mandibular ramus. (E) Frontal and (F) left lateral photos of the patient's dental models mounted on an articulator. (G) The lower cast was segmented through the symphysis and the pretrauma occlusion was re-established (H,I). Intraoperative photographs of the symphysis (J) after an osteotomy was performed through the original fracture site and the left angle (K) after a sagittal ramus osteotomy was performed through the ramus, fragment mobilization, reestablishment of mandibulomaxillary fixation, and bone plate osteosynthesis. (L) Postoperative panoramic radiograph showing completed osteotomies. (M) Frontal photograph of the patient after healing. (N) Occlusal photograph after healing and arch-bar removal. 
teeth, and improper application of compression techniques can very easily lead to healing in the wrong position.

One must pay special attention to assure that the lingual cortex has been adequately reduced in a symphyseal fracture because the intraoral approach usually hides the lingual cortex from the surgeon, creating a false sense of adequate reduction when evaluating the buccal cortex only. To assure this does not occur, one must use manual pressure applied to the mandibular angles until the point where the fractured buccal cortices separate a bit, assuring proper lingual adaptation. Alternatively, one can surgically expose the lingual cortex by different surgical exposure techniques. ${ }^{16}$

Inadequate adaptation of a bone plate can cause malunion. The precise adaptation of a bone plate to the underlying bone is never really known with any certainty until the screws (nonlocking) have been inserted and tightened. If the plate was not properly adapted, the bone fragments will not be properly reduced, and this may cause a malocclusion. The use of locking screw/plate systems has helped eliminate this problem because the bone is not drawn toward the plate when the screws are tightened.

Failure to establish the pretrauma occlusion is another common cause of malunion and malocclusion. ${ }^{16}$ Asking the patient or their family for a history of prior orthodontic therapy and/or the acquisition of dental models as well as pretrauma photographs can be very useful to figure out what the pretrauma occlusion was like.

A frequent cause of malocclusion is from condylar process fractures. One must understand that closed treatment of condylar process fractures accepts a malposition of the condylar fragment, resulting in a malunion. However, most of the time with proper rehabilitation, a malocclusion can be prevented. When closed treatment is not successful, a malocclusion results. Similarly, with open treatment of condylar process fractures, the condylar fragment must be properly reduced prior to application of internal fixation hardware to assure the occlusion is restored.

Treatment of malunions necessitates identification of the cause and assessment of the severity. If the malocclusion is minor, occlusal adjustments by grinding the teeth and/or orthodontic/orthopedic means can be attempted for a short period of time. Many minor occlusal irregularities will be satisfactorily treated by elastic traction between the upper and lower teeth. For more severe malunions, osteotomies may be necessary. In such instances, the old fracture site may serve as the site of osteotomy. In others, more standard osteotomy sites commonly used in orthognathic surgery can be employed. An orthognathic surgery work-up will help.

\section{Fibrous Union/Nonunion}

The lack of osseous healing after an adequate period of time (usually 6 months in long bones and 12 weeks in the mandible) leads to fibrous union. ${ }^{16}$ Diagnosis of fibrous union is usually made clinically by detecting mobility across the site of fracture. This mobility can be painful to the patient and present itself with or without infection (-Fig. 2). Sometimes infection is the consequence of a smaller fragment that is loose and working its way out of the body.

The most common causes of fibrous union are fracture instability, early infection, and inaccurate reduction with
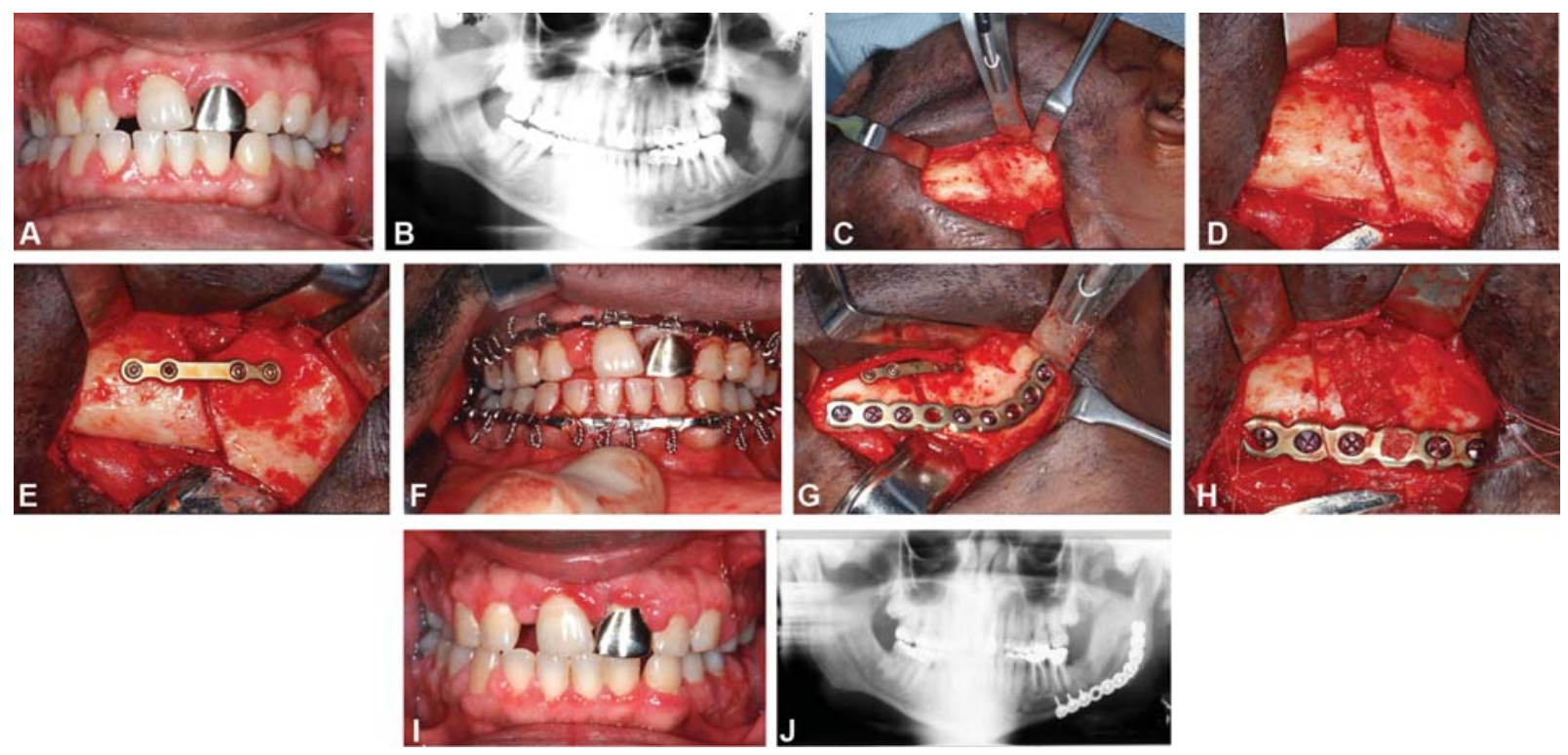

Fig. 2 Records of a patient who underwent extraction of a lower left third molar and subsequently developed a fracture through the mandibular angle. The patient was placed into mandibulomaxillary fixation (MMF) for several weeks, but the fracture never consolidated. The patient had slight mobility and pain when occluding. (A) Photograph of slight malocclusion caused by the fracture. (B) Panoramic radiograph showing fracture through the left angle. Note patient is in MMF using lvy loops. (C) The fracture was exposed through a transfacial approach. Note the fibrous tissue within the fracture gap. (D) The fracture after removal of the fibrous tissue and mobilization. (E) Temporary fixation after the patient was placed into MMF and the condylar fragment seated into the glenoid fossa. (F) Intraoperative check of the occlusion by taking out of MMF and assuring that the mandibular dentition rotates into the proper relationship with the maxillary dentation. (G) Final load-bearing fixation applied across the fracture site. $(\mathbf{H})$ Temporary fixation plate is removed and particular bone marrow graft is inserted into the fracture site. (I) Final occlusal relationship after healing. (J) Final panoramic radiograph demonstrating osseous healing. 
lack of osseous contact between the fragments. Inadequate hardware selection, for example, a bone plate that is too small or not enough screws per side can lead to mobility and nonunion. Similarly, loosening of the bone screws from the bone can lead to fracture mobility.

Postoperative infections leading to bone "softening" due to the acidic environment induced by the bacteria can cause loosening of fixation devices, sequestration of bone, and eventually mobility of the fracture. ${ }^{16}$

Inaccurate reduction of the fragments resulting in a diminished area of osseous contact makes mobility of the fragments more likely and fibrous deposition inevitable. This is especially true for fractures through the atrophic mandibular body area in the elderly.

Bone grafting is rarely needed when treating mandible fractures. Due to the rich vascularity of the face, healing is usually possible even in the presence of a contaminated environment (oral cavity) and a difficult group of noncomplaint patients.

There are some indications for immediate bone grafting and reconstruction to promote adequate healing. Mandibles with a cross-sectional area of $1^{2} \mathrm{~cm}$ or less often leave very little bone in contact. It is not surprising that the fibrous union rate for atrophic fractures is often reported as over $20 \%{ }^{16,17}$ Atrophic mandibles, especially in the elderly, should be grafted to promote healing and decrease the rate of nonunion. Price and Ellis advocate primary reconstruction of atrophic mandible fractures using reconstruction plates and autogenous bone grafts for such fractures. ${ }^{18}$ In selected cases in which multiple comorbidities may influence local and/or systemic outcomes, bone morphogenetic proteins (BMPs) and tricalcium phosphate can be used as an alternative to autogenous grafts. ${ }^{16,17,19}$

The treatment of fibrous union requires identification of the cause and its treatment. If there is an active infection, the infection must be controlled. Extraction of any de-vital teeth in the line of fracture and debridement of any necrotic soft and hard tissue and restabilization of the fracture must occur. $^{20,21}$ In most instances, especially those that are long-standing fibrous unions, bone grafting will be necessary to effect osseous union. The fragments must be exposed and cleaned of any fibrous tissue. After the fragments have been freshened, the proper occlusal relationship must be reestablished by maxillomandibular fixation (MMF). If there are no teeth posterior to the fracture gap, the ramal segment must be manipulated to assure placement of the mandibular condyle into the mandibular fossa. The fracture gap will then usually appear to be greater than originally noted before reestablishment of the occlusion. The segments are then stabilized by a reconstruction bone plate (load-bearing fixation), assuring passive placement on the segments. There should be at least four screws on each side of the fracture gap, and no screw should be closed than $7 \mathrm{~mm}$ to the fracture gap. The bone may appear normal, but there is less mineral content for several millimeters from the fracture. Placement of the screws away from the fracture gap will assure stable fixation. The MMF should then be removed to verify proper occlusion. Autogenous particulate bone graft, BMP, or any other growth factors can be considered and packed into the defect; the soft tissues are then closed in layers.

\section{Infection}

Infections are one of the most common complications of mandibular fracture management, irrespective of how the fracture was treated ( $\mathbf{- F i g} . \mathbf{3})^{1-3}$ They tend to be more common when fractures are treated open, but this may be due to the more complex cases usually requiring open treatment. The oral cavity is a reservoir for bacteria that can easily colonize the surgical site or internal fixation hardware. The difference between infection and osteitis is that osteitis has no great component of bacterial cellulitis and no abscess formation or purulent discharge associated with it. Osteitis is an osteomyelitis that is localized and is due to devitalization of the bone from traumatic and/or surgical
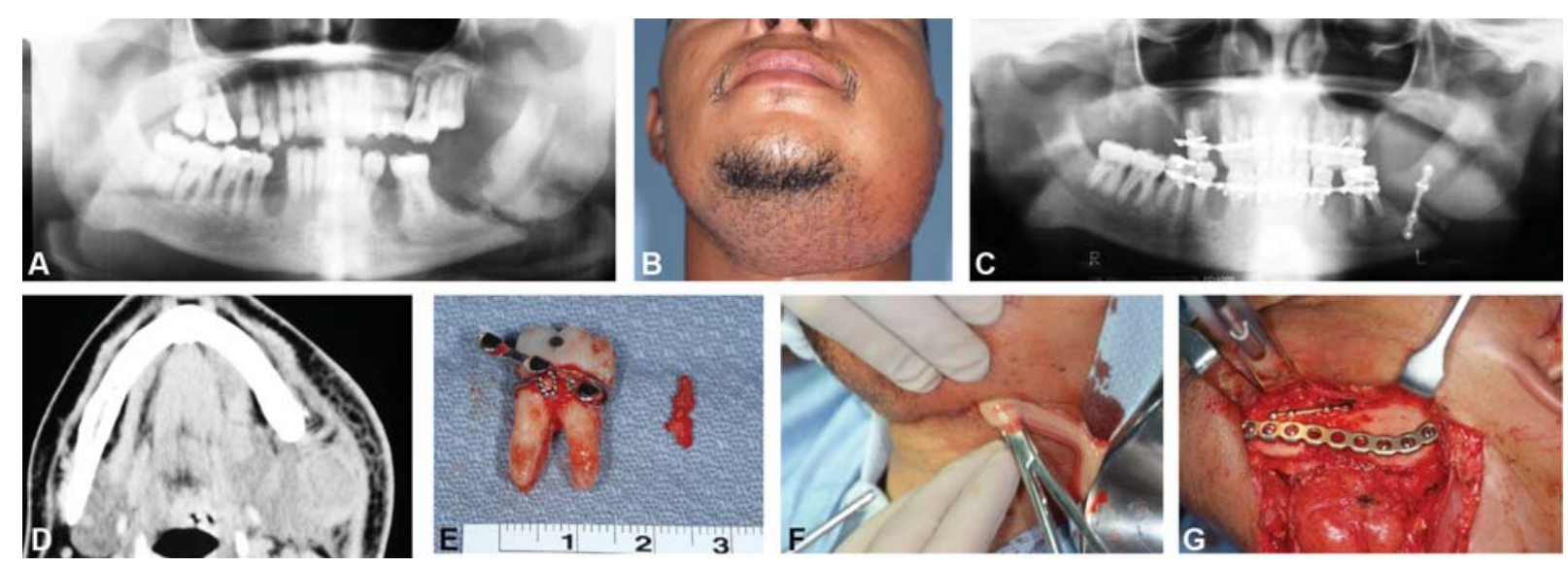

Fig. 3 (A) panoramic radiograph of a patient with a left angle fracture. It was treated with a single miniplate. (B) Facial photograph 6 weeks after surgery showing redness and swelling of the left angle region. (C) Panoramic radiograph showing unstable fixation and clockwise rotation of the mandibular ramus on the left side. (D) Computed tomography scan showing large abscess formation around the left angle of the mandible. The patient was taken to surgery and the left miniplate was removed. The left lower molar was also removed (E) because it was infected, and an incision and drainage was performed through a transfacial approach (F). A reconstruction bone plate was applied at the same time ( $G)$ and a drain was placed. (H) Panoramic radiograph at 12 weeks showing complete healing. 
disruption of superficial blood supply. The fracture may be completely stable with osteitis or infection, but infection is more likely to be associated with fracture instability.

Fracture instability can also lead to infection. When mobility is present during the early stages of healing, disruption of blood supply occurs, and the interference in revascularizing leads to devitalization of bone. The presence of mobility and/or devitalization of bone with microorganisms results in infection of the fracture.

Treatment of infection requires assessment of the stability of the fracture. If the fracture was treated closed and a postoperative infection occurred, one should determine whether or not the MMF provides stable fixation or not. Other causes of the infection must also be sought such as a devital tooth in the line of the fracture. If the fracture were treated open with internal fixation devices, an assessment of whether or not the hardware is continuing to provide stability to the fracture is imperative. If the fracture is stable and there is no evidence of a loose screw or plate, management of the infection is indicated without removal of the hardware. Incision and drainage and irrigations, systemic antibiotics, treatment or extraction of devital teeth, debridement of devital bone, and systemic management of the patient's general health are indicated. Any loose hardware must be removed because it tends to perpetuate infections. If removal of the hardware is necessary, the fracture must be restabilized. To provide stabilization, one has several options. One can use MMF, external pin fixation, or restabilization with internal fixation devices. If the latter course is chosen, the fracture should be stabilized by very stable means such as by using a reconstruction bone plate (load-bearing fixation) with at least four screws on each side of the fracture. The screws should be kept at least $7 \mathrm{~mm}$ away from the fracture. One may wish to place irrigation drains and continue irrigations until they clear. ${ }^{16}$

Teeth in the line of fracture must be removed when they preclude the correct reduction of the segments or if they represent a risk for infection. Teeth with fractured roots, extensive periodontal damage, or any periapical damage must be removed. One must consider the design of the

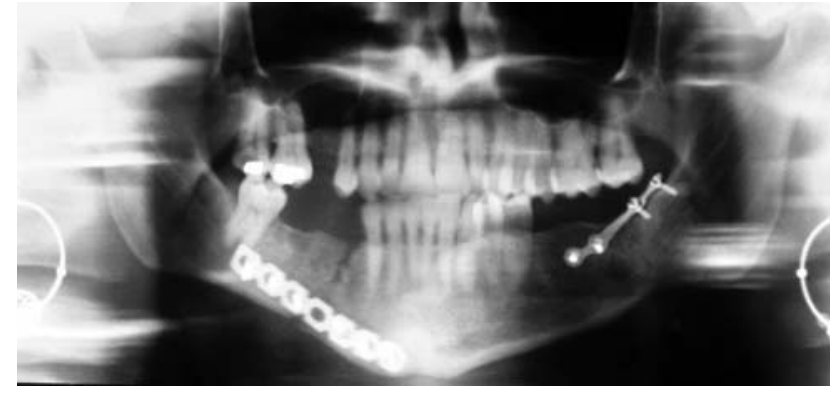

Fig. 5 Postoperative panoramic radiograph of a patient who was treated with bone plate fixation for a left angle and right body fracture. Both were treated through a transoral approach. Note that the posterior portion of the reconstruction bone plate applied to the right mandibular body is too high, with all screws entering the inferior alveolar canal. This can occur because visibility and access in the posterior mandible is very limited when using a transoral approach.

flap anytime an extraction is planned to allow for adequate coverage of the hardware used. ${ }^{20,21}$

According to Chrcanovic, tooth buds in the line of pediatric mandibular fractures should not be removed or replaced in the (alleged) proper position despite the degree of displacement, as studies showed that even tooth buds in the early stage of calcification and those involved in widely displaced fracture sites continued development and erupt. ${ }^{22}$ Intact teeth should also be left in situ if they show no evidence of severe loosening or inflammatory change. Permanent teeth maintained in the line of fracture should be followed up clinically and radiographically for at least 1 year to assess for the need of endodontic treatment. ${ }^{22}$

\section{latrogenic Complications}

The most common iatrogenic complication that can occur when rigid internal fixation of mandibular fractures is used is placement of a screw or screw hole through a normal anatomical structure such as a tooth root or the mandibular neurovascular bundle ( $\boldsymbol{- F i g s . ~} 4$ and $\mathbf{5}$ ). Because the mandible contains tooth roots above and the inferior alveolar neurovascular canal in the middle, the only place where bicortical
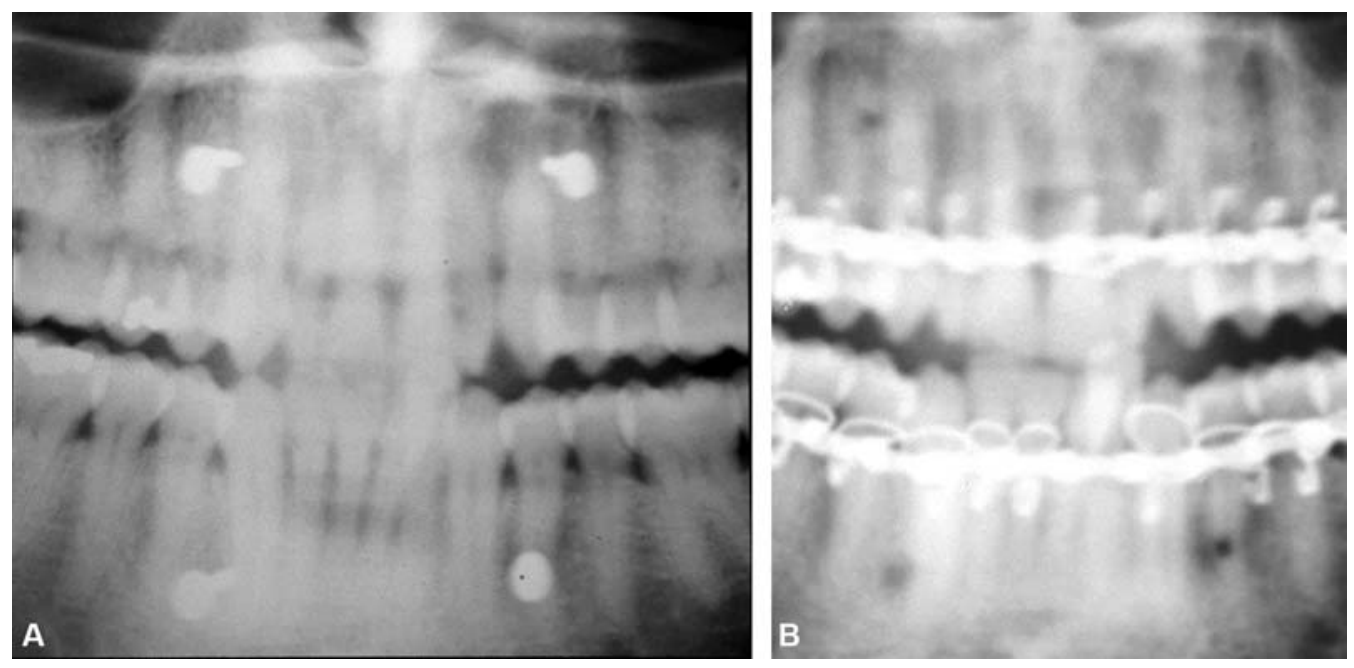

Fig. 4 Radiographs of a patient whose mandibular fracture was stabilized with mandibulomaxillary fixation screws. (A) Immediately after surgery. (B) After removal of the MMF screws. Note the hole through the root of the lower left first premolar. 
bone plates can be applied safely on the lateral cortex is along the inferior border.

Placement of a screw hole or screw through the mandibular canal or tooth root might be obvious if witnessed during surgery but may not be apparent until the postoperative period. If there is radiographic evidence of a screw through the mandibular canal combined with a postoperative neurosensory test positive for dysfunction that was not present in the preoperative exam, one should assume that an injury has occurred.

If the injury is witnessed during drilling, one should not insert the screw. The fixation device should be repositioned, and new holes drilled. If not witnessed but strongly suspected, postsurgery images must be obtained, the patient must be informed, and a decision should be made to either remove the screw after the fracture heals or remove the screw now and use alternate means of fixation. The regenerative ability of the mandibular nerve inside an intact canal varies, but, usually, extensive surgery to expose and repair is not advocated. Regeneration will have a fair chance, depending on other systemic and local factors and the age of the patient.

The consequences of drilling a hole through a tooth root are usually not catastrophic as long as a screw is not placed. Studies in adult monkeys have shown that there is collateral circulation in the dental pulp, and transection of one root apex does not always devitalize a multirooted tooth. ${ }^{23}$ These findings should not make the surgeon cavalier about where holes are drilled in the mandible. However, they may help direct subsequent management of the tooth.

\section{Complications Related to the Surgical Approach}

The most common anatomical injury when treating mandibular fractures is damage to the trigeminal nerve (CN V). The mandibular canal must be avoided when placing rigid internal fixation devices. Injuries usually occur when placing a bicortical screw in the posterior body/angle region through an intraoral approach using transbuccal instrumentation. Inadequate access and visibility and/or inadequate familiarity with mandibular anatomy is usually the reason. The other area is the region of the mental nerve (-Fig. 6). Parasym-

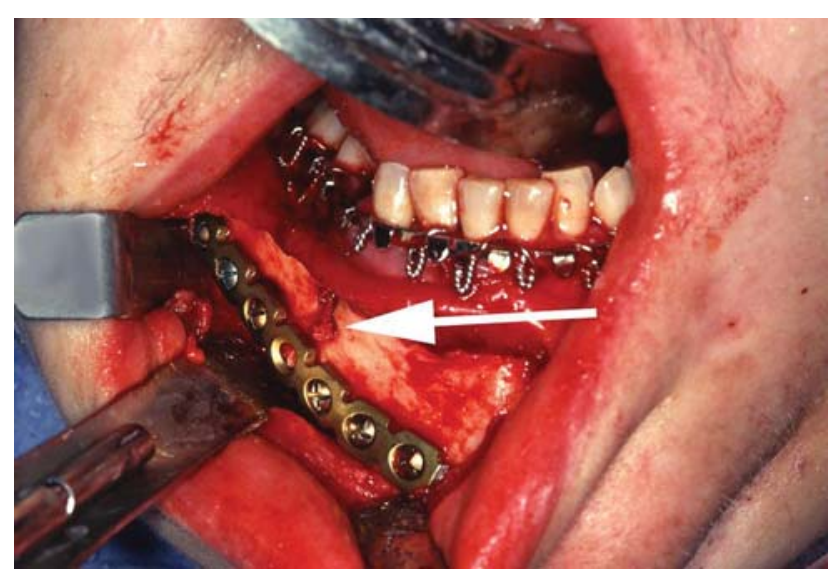

Fig. 6 Intraoperative photograph of a patient treated through a transoral approach for a right mandibular body fracture. Note that the mental nerve has been avulsed. physeal fractures often require exposure of the mental nerve and skeletonization of the nerve bundle to allow for better retraction. It is paramount to perform an excellent presurgical examination to document the degree of any preexisting injury to the nerve.

Most injuries to $\mathrm{CN} \mathrm{V}$ heal and patients recover their sensation. Visualized transections can be repaired using a direct approximation technique if the fascicles are intact or by using a nerve graft and connector-assisted techniques. ${ }^{24,25}$

Facial nerve injuries are most common when choosing an extraoral approach to the mandible. These injuries are less than $2.5 \%$ in most studies, ${ }^{26}$ although they approach $20 \%$ for transfacial surgical approaches to the condylar process. ${ }^{26}$ The anesthesiologist can be asked to use reversible muscle relaxants to allow monitoring the course of the nerve to help minimize the chance of nerve injury when using an extraoral approach. Fortunately, most CN VII injuries are of only one or two branches, such as the marginal mandibular and/or buccal branches, and patients adapt to these without much dysfunction.

\section{Comorbidities and Antibiotics}

It is well known that uncontrolled diabetes, smoking, and alcoholism increase the risk of infection and decrease the healing rate of most surgical procedures. ${ }^{1-4,27}$ Furr et al found a significant positive correlation between tobacco, alcohol, drug use, and long-term complications. ${ }^{1}$ When treating mandible fractures, most clinicians will use antibiotics. The rationale is that fractures are exposed to the oral cavity through the periodontal ligament, making these compound contaminated fractures. Preoperative antibiotics have been shown to significantly reduce the number of postoperative infections. ${ }^{28}$ Routine use of postoperative antibiotics when treating uncomplicated mandibular fractures has been examined in the literature and, and although it is recommended by multiple authors, is not supported by the current evidence. ${ }^{29,30}$

\section{Trauma-Induced Temporomandibular Disorders}

Temporomandibular disorders (TMDs) are very common in developed countries and are multifactorial in cause. ${ }^{31}$ Stress, anxiety, malocclusion, internal derangements, muscle spasms, and eating and functional habits among others have been identified as important factors in TMDs. Such factors can be considered as causing microtrauma to the joint. Condylar or noncondylar mandibular fractures would be considered forms of macrotrauma to the temporomandibular joint (TMJ) and have been implicated as an important factor with intraarticular TMDs. ${ }^{31}$ Animal studies have shown that condylar trauma results in biochemical changes within the TMJ, cartilage degeneration, and intraarticular adhesions. ${ }^{31}$ The long-term effect of such TMJ changes on jaw function is not clear, and although most clinicians are aware of the association between mandibular trauma and the later development of TMD, there have been few systematic studies. When one considers how common TMDs are in the general population and how relatively rare macrotrauma to the TMJ is, it is hard to implicate trauma as a major cause of TMDs. ${ }^{31}$

Most patients respond well to TMJ trauma and adapt with very few complaints in the long term. However, it is important 
to realize that trauma may play an important role in the onset of acute TMDs or may exacerbate an already preexistent and perhaps dormant TMD.

TMJ trauma has been implicated as an important etiological factor in TMJ ankylosis, especially in untreated or poorly treated patient populations. While the most common cause of TMJ ankylosis is condylar fracture, the incidence of TMJ ankylosis from condylar fracture is very low $(<0.05 \%) .^{31,32}$

\section{Conflict of Interest \\ None declared.}

\section{References}

1 Furr AM, Schweinfurth JM, May WL. Factors associated with longterm complications after repair of mandibular fractures. Laryngoscope 2006;116(03):427-430

2 Christensen BJ, Mercante DE, Neary JP, King BJ. Risk factors for severe complications of operative mandibular fractures. J Oral Maxillofac Surg 2017;75(04):787.e1-787.e8

3 Odom EB, Snyder-Warwick AK. Mandible fracture complications and infection: the influence of demographics and modifiable factors. Plast Reconstr Surg 2016;138(02):282e-289e

4 Rashid S, Kundi JA, Sarfaraz A, Qureshi AU, Khan A. Patterns of mandibular fractures and associated comorbidities in Peshawar, Khyber Pakhtunkhwa. Cureus 2019;11(09):e5753

5 James J, Farrell T IV, Stevens M, Looney S, Faigen A, Anderson J. Time to open repair of mandibular fractures and associated complications. J Oral Maxillofac Surg 2020;78(01):101-107

6 Lamphier J, Ziccardi V, Ruvo A, Janel M. Complications of mandibular fractures in an urban teaching center. J Oral Maxillofac Surg 2003;61(07):745-749, discussion 749-750

7 Haug RH, Schwimmer A. Fibrous union of the mandible: a review of 27 patients. J Oral Maxillofac Surg 1994;52(08):832-839

8 Bochlogyros PN. Non-union of fractures of the mandible. J Maxillofac Surg 1985;13(04):189-193

9 Champy M, Loddé JP, Schmitt R, Jaeger JH, Muster D. Mandibular osteosynthesis by miniature screwed plates via a buccal approach. J Maxillofac Surg 1978;6(01):14-21

10 Cawood JI. Small plate osteosynthesis of mandibular fractures. Br J Oral Maxillofac Surg 1985;23(02):77-91

11 Lucca M, Shastri K, McKenzie W, Kraus J, Finkelman M, Wein R. Comparison of treatment outcomes associated with early versus late treatment of mandible fractures: a retrospective chart review and analysis. J Oral Maxillofac Surg 2010;68(10):2484-2488

12 Lee UK, Rojhani A, Herford AS, Thakker JS. Immediate versus delayed treatment of mandibular fractures: A stratified analysis of complications. J Oral Maxillofac Surg 2016;74(06):1186-1196

13 Barker DA, Oo KK, Allak A, Park SS. Timing for repair of mandible fractures. Laryngoscope 2011;121(06):1160-1163

14 Hermund NU, Hillerup S, Kofod T, Schwartz O, Andreasen JO. Effect of early or delayed treatment upon healing of mandibular fractures: a systematic literature review. Dent Traumatol 2008;24 $(01): 22-26$
15 Stone IE, Dodson TB, Bays RA. Risk factors for infection following operative treatment of mandibular fractures: a multivariate analysis. Plast Reconstr Surg 1993;91(01):64-68

16 Ellis E III. Complications of rigid internal fixation for mandibular fractures. J Craniomaxillofac Trauma 1996;2(02):32-39

17 Bruce R, Fonseca RJ. Mandibular fractures. In: Fonseca RJ, Walker RV, eds. Oral and Maxillofacial Trauma. Philadelphia, PA: W.B. Saunders; 1991:359-417

18 Ellis E, Price C. Treatment protocol for fractures of the atrophic mandible. J Oral Maxillofac Surg 2008;66:421-435

19 Castro-Núñez J, Cunningham LL, Van Sickels JE. Atrophic mandible fractures: are bone grafts necessary? An update. J Oral Maxillofac Surg 2017;75(11):2391-2398

20 Aulakh KK, Gumber TK, Sandhu S. Prognosis of teeth in the line of jaw fractures. Dent Traumatol 2017;33(02):126-132

21 Taysi M, Yildirim S. Should the teeth in the line of jaw fractures be extracted? J Istanb Univ Fac Dent 2015;49(01):61-65

22 Chrcanovic BR. Teeth in the line of mandibular fractures. Oral Maxillofac Surg 2014;18(01):7-24

23 Hitchcock R, Ellis E III, Cox CF. Intentional vital root transection: a 52-week histopathologic study in Macaca mulatta. Oral Surg Oral Med Oral Pathol 1985;60(01):2-14

24 Zuniga JR, Williams F, Petrisor D. A case-and-control, multisite, positive controlled, prospective study of the safety and effectiveness of immediate inferior alveolar nerve processed nerve allograft reconstruction with ablation of the mandible for benign pathology. J Oral Maxillofac Surg 2017;75(12):2669-2681

25 Yampolsky A, Ziccardi V, Chuang SK. Efficacy of acellular nerve allografts in trigeminal nerve reconstruction. J Oral Maxillofac Surg 2017;75(10):2230-2234

26 Al-Moraissi EA, Louvrier A, Colletti G, et al. Does the surgical approach for treating mandibular condylar fractures affect the rate of seventh cranial nerve injuries? A systematic review and meta-analysis based on a new classification for surgical approaches. J Craniomaxillofac Surg 2018;46(03):398-412

27 Lassig AAD, Bechtold JE, Lindgren BR, et al. Tobacco exposure and wound healing in head and neck surgical wounds. Laryngoscope 2018;128(03):618-625

28 Zallen RD, Curry JT. A study of antibiotic usage in compound mandibular fractures. J Oral Surg 1975;33(06):431-434

29 Abubaker AO, Rollert MK. Postoperative antibiotic prophylaxis in mandibular fractures: A preliminary randomized, double-blind, and placebo-controlled clinical study. J Oral Maxillofac Surg 2001; 59(12):1415-1419

30 Bormann KH, Wild S, Gellrich NC, et al. Five-year retrospective study of mandibular fractures in Freiburg, Germany: incidence, etiology, treatment, and complications. J Oral Maxillofac Surg 2009;67(06):1251-1255

31 Al-Moraissi EA, Perez D, Ellis E III. Do patients with malocclusion have a higher prevalence of temporomandibular disorders than controls both before and after orthognathic surgery? A systematic review and meta-analysis. J Craniomaxillofac Surg 2017;45(10): 1716-1723

32 Anyanechi CE. Temporomandibular joint ankylosis caused by condylar fractures: a retrospective analysis of cases at an urban teaching hospital in Nigeria. Int J Oral Maxillofac Surg 2015;44 (08):1027-1033 